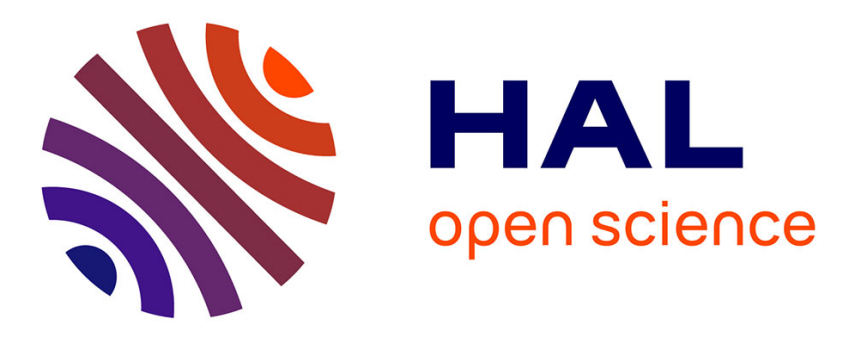

\title{
CDMA-based multiple multicast communications on WiNOC for efficient parallel computing
}

Navonil Chatterjee, Hemanta Kumar Mondal, Rodrigo Cataldo, Jean-Philippe Diguet

\section{- To cite this version:}

Navonil Chatterjee, Hemanta Kumar Mondal, Rodrigo Cataldo, Jean-Philippe Diguet. CDMAbased multiple multicast communications on WiNOC for efficient parallel computing. 13th IEEE/ACM International Symposium on Network On Chip, Oct 2019, New York, United States. 10.1145/3313231.3352371. hal-02408590

\section{HAL Id: hal-02408590 \\ https://hal.science/hal-02408590}

Submitted on 8 Dec 2020

HAL is a multi-disciplinary open access archive for the deposit and dissemination of scientific research documents, whether they are published or not. The documents may come from teaching and research institutions in France or abroad, or from public or private research centers.
L'archive ouverte pluridisciplinaire HAL, est destinée au dépôt et à la diffusion de documents scientifiques de niveau recherche, publiés ou non, émanant des établissements d'enseignement et de recherche français ou étrangers, des laboratoires publics ou privés. 


\section{CDMA-based Multiple Multicast communications on WiNOC for efficient parallel computing}

\author{
Navonil Chatterjee \\ chatterjee.navonil@univ-ubs.fr \\ Lab-STICC, UBS \\ Rodrigo Cataldo \\ rodrigo.cataldo@acad.pucrs.br
}

Lab-STICC, UBS

\begin{abstract}
In this work, we introduce an hybrid WiNoC, which judicially uses the wired and wireless interconnects for broadcasting/multicasting of packets. A code division multiple access (CDMA) method is used to support multiple broadcast operations originating from multiple applications executed on the multiprocessor platform. The CDMAbased WiNoC is compared in terms of network latency and power consumption with wired-broadcast/multicast NoC.
\end{abstract}

\section{CCS CONCEPTS}

- Networks $\rightarrow$ Network on chip; $\bullet$ Computer systems organization $\rightarrow$ Interconnection architectures; $\bullet$ Computing methodologies $\rightarrow$ Parallel computing methodologies.

\section{KEYWORDS}

Wired/Wireless Network-on-Chip, Broadcast and Multicast operation, Parallel computing, Code Division Multiple Access (CDMA)

\section{INTRODUCTION}

WiNoC has emerged as a viable solution for the implementation of broadcast [1] communications on large manycores. Also, multiple applications can be executed simultaneously on a given WiNoC platform designed for large manycore architectures. In such a case, broadcast operation in one application may interfere with another one running in the same WiNoC, which might lead to contention and increase in power consumption as a single wireless channel is used by multiple application for broadcast communication.

In this work, we propose an original strategy to mitigate the aforementioned problems. We present a WiNoC topology which is divided into uniform clusters where each cluster is associated with one wireless interface. As, all the PEs are not associated with wireless interface, we use hybrid mechanism where both wired and wireless links are used for broadcast and multicast operations. For unicast packet transmission, only wired NoC is used. We have used code division multiple access (CDMA) based wireless packet

\author{
Hemanta Kumar Mondal \\ hemanta.mondal@ece.nitdgp.ac.in \\ NIT Durgapur \\ Jean-Philippe Diguet \\ jean-philippe.diguet@univ-ubs.fr \\ Lab-STICC, CNRS
}

transmission to support parallel broadcast communication in two or more applications executing in a WiNoC based multiprocessor platform. The proposed method helps to improve the overall system performance by providing efficient broadcast/multicast communication for single and multiple applications.

\section{SYSTEM ARCHITECTURE}

We consider a 2D Mesh based Wireless NoC which is divided into equal sized clusters as shown in Figure 1a. Each cluster consists of 16 routers which are arranged in $4 \times 4$ fashion. Every cluster has a centrally placed wireless hub with a wireless interface (WI) providing inter-cluster wireless links. The wireless hub is connected to one router in the cluster, which is known as hub connection (HC) router. Thus, all packets communicated using the wireless hub, flow though the $\mathrm{HC}$ router. For unicast communication, we have used table based routing while broadcast packets are communicated using WHIRL routing algorithm [3].

The Wireless Interface (WI) connects the router with the wireless medium. The WI consists of 3 parts: (1) Antenna, (2) Analog domain and (3) Digital domain, as shown in Figure 1b. The digital domain consists of Serializer/ Deserializer, CDMA encoder/decoder and channel access controller. The main component of the analog domain is the On-Off Keying (OOK) transceiver module. zigzag metal antenna is used for signal transmission and reception. A MAC mechanism is used to share wireless channels among multiple users. We based our choice on a contention free MAC protocol as used in OrthoNoC[1] and modified it to suit our requirements.

\section{BROADCAST \& MULTICAST OPERATIONS}

In this work, we have assumed that a single application or multiple applications may be executed in parallel on the multicore WiNoC. In case of single application, during broadcast operation only one hub works as the source and all other hubs as the destination. There is no conflict with another broadcast, if two of them do not start at the same time. Thus no code is required as all the hubs receive the message. However, in case of multiple applications are executed in parallel, a conflict may occur if both the applications launch a broadcast operation at the same time. In such as case, different CMDA codes will be assigned to the applications. In this work, we have used two type of CMDA based code-channel: (i) two codechannels and (ii) four code-channels, where maximum of two and four applications are executed in parallel, respectively.

\section{RESULTS \& DISCUSSION}

To analyze the performance of the proposed CDMA-based multicast, we have used the Noxim [2] network-on-chip simulator. We 


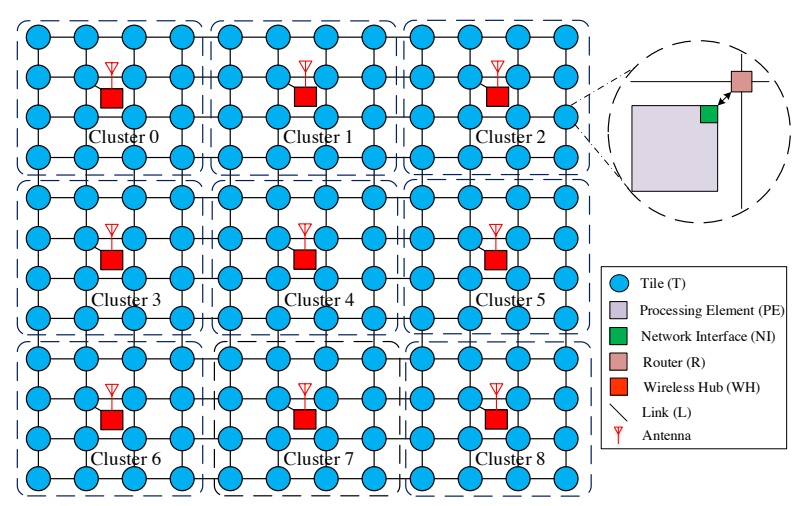

(a) $12 \times 12$ Wireless NoC

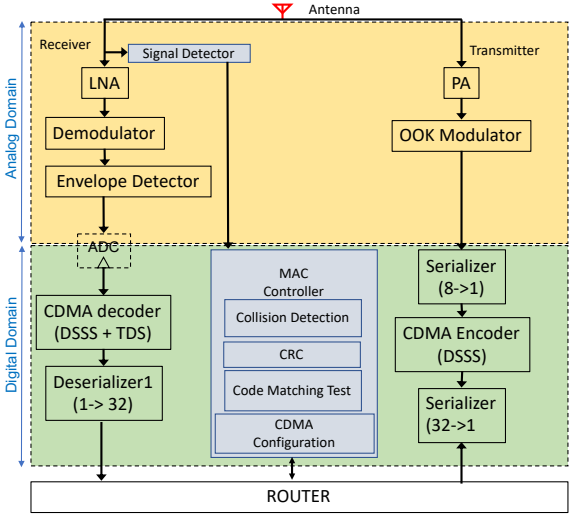

(b) Wireless Interface Architecture

Figure 1: Overview of the system Model

have used PARSEC benchmark applications, bodytrack and streamcluster to analyze broadcast operations in WiNoC. To evaluate of the multicast operations, synthetic applications are generated and combined to form 4 different scenarios. We have compared our proposed technique (WiNoC) with wired broadcast/multicast scheme denoted as NoC+WHIRL [3]. To reduce the power consumption of the hubs, power gating is used in LNA and PA when hubs are not used as described in [4].

The network latency is presented in Fig 2(a). Compared to wired broadcast (NoC+WHIRL), we observe that our proposed method provides $29.11 \%, 19.81 \%$ and $28.48 \%$, improvement in network latency for Bodytrack(med), Bodytrack(lar) and Streamcluster(med), respectively. Figure 2(b) depicts the power delay product (PDP) for NoC+WHIRL and the proposed WiNoC for different benchmark applications.When compared with NoC+WHIRL, we observe that the propose method achieves $38.8 \%, 30.7 \%$, and $38.2 \%$ improvement in PDP for Bodytrack(med), Bodytrack(lar) and Streamcluster(med).
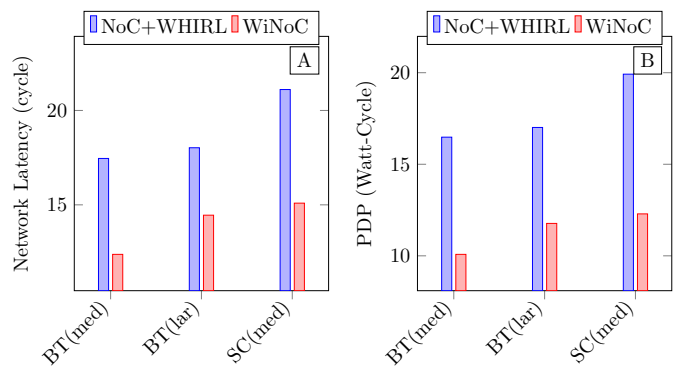

Figure 2: Analysis of (a) Network Latency and (b) PDP for broadcast operation using different benchmark applications

Figure 3(a) reports the latency for different scenarios. Compared to NoC+WHIRL, CDMA-based WiNoC shows $21.01 \%, 20.8 \%, 19 \%$ and 5\% improvement in network latency for scenario 1, 2, 3 and 4 respectively. The power delay product for $\mathrm{NoC}+\mathrm{WHIRL}$ and proposed WiNoC for different scenarios is presented in Figure 3(b). Comparison with NoC+WHIRL, there is $4.25 \%, 4.12 \%$, and $2.65 \%$ improvement in PDP for scenario 1, scenario 2 and scenario 3, respectively. In scenario 4, we observe a degradation in PDP.
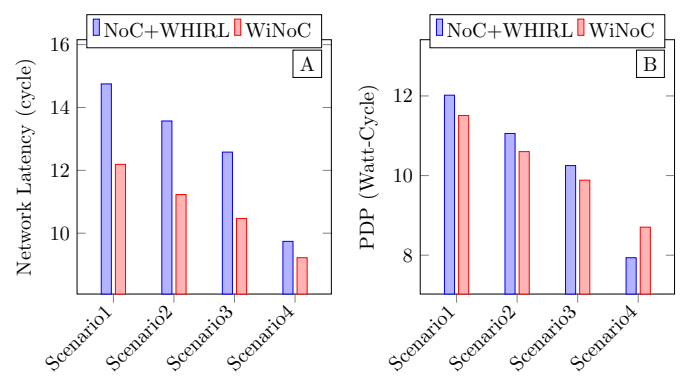

Figure 3: Analysis of (a) Network Latency and (b) PDP for multicast operation using different synthetic applications

\section{CONCLUSION}

In this paper, we present a solution to efficiently implement broadcast and multicast operations on future large manycore architecture with the proposed Wireless NoC. We have implemented a CDMAbased transmission protocol for multicast operations in parallel applications. The proposed WiNoC supports maximum of four code-channels which allows execution of four parallel applications.

\section{ACKNOWLEDGEMENT}

This work is a part of BBC project supported by Labex CominLABS.

\section{REFERENCES}

[1] S. Abadal, J. Torrellas, E. AlarcÃşn, and A. Cabellos-Aparicio. 2018. OrthoNoC: A Broadcast-Oriented Dual-Plane Wireless Network-on-Chip Architecture. IEEE Transactions on Parallel and Distributed Systems 29, 3 (March 2018), 628-641.

[2] Vincenzo Catania, Andrea Mineo, Salvatore Monteleone, Maurizio Palesi, and Davide Patti. 2017. Improving Energy Efficiency in Wireless Network-on-Chip Architectures. 7. Emerg. Technol. Comput. Syst. 14, 1, Article 9 (Nov. 2017), 24 pages.

[3] Tushar Krishna, Li-Shiuan Peh, Bradford M. Beckmann, and Steven K. Reinhardt. 2011. Towards the Ideal On-chip Fabric for 1-to-many and Many-to-1 Communication. In Proceedings of the 44th Annual IEEE/ACM International Symposium on Microarchitecture (MICRO-44). ACM, New York, NY, USA, 71-82.

[4] H. K. Mondal, R. C. Cataldo, C. A. Missio Marcon, K. Martin, S. Deb, and J. Diguet. 2018. Broadcast- and Power-Aware Wireless NoC for Barrier Synchronization in Parallel Computing. In 2018 31st IEEE International System-on-Chip Conference (SOCC). 1-6. 